\title{
MONITORAMENTO POR ESPECTROFOTOMETRIA DO TRATAMENTO DE MANIPUEIRA VIA REAÇÃO DE FENTON
}

\author{
J. C. ZENI ${ }^{1}$, G. V. LEON $^{1}$, V. LEIFELD ${ }^{2}$ e L. IGARASHI-MAFRA ${ }^{1,2}$ \\ ${ }^{1}$ Universidade Federal do Paraná, Departamento de Engenharia Química \\ ${ }^{2}$ Universidade Federal do Paraná, Programa de Pós-Graduação em Engenharia de Alimentos \\ E-mail para contato: ju_zeni@yahoo.com.br
}

\begin{abstract}
RESUMO - A manipueira é um efluente derivado do processamento de farinha e fécula de mandioca. Devido a sua elevada carga orgânica, atribuída à presença de altas concentrações de carboidratos, causa um impacto ambiental muito grande. Processos convencionais de tratamento são ineficientes e, assim, o objetivo deste trabalho foi avaliar as reações de Fenton via espectrofotometria UV-Vis para a degradação da matéria orgânica da manipueira. Primeiramente foi feita a caracterização do efluente bruto com relação a diferentes parâmetros físicoquímicos. Como pré-tratamentos, foram realizados a coagulação/floculação, seguido de tratamento biológico por sistema de lodos ativados. Em seguida, foram feitos os ensaios de Fenton, com diferentes $\mathrm{pH}(2,8 ; 3,0$ e 3,2), concentrações de peróxido de hidrogênio $\left(10,15\right.$ e $\left.20 \mathrm{~g} \mathrm{~L}^{-1}\right)$ e de ferro $\left(60,180\right.$ e $\left.300 \mathrm{mg} \mathrm{L}^{-1}\right)$ de acordo com as combinações obtidas em planejamento experimental $3^{2}$. Como resultado, pôde-se verificar que as menores relações A/A0 (parâmetro de avaliação da degradação) foram obtidas em $\mathrm{pH} 3,0,\left[\mathrm{H}_{2} \mathrm{O}_{2}\right]$ de $15 \mathrm{~g} \mathrm{~L}^{-1}$ e Fe $^{2+} 180$ $\mathrm{mg} \mathrm{L}^{-1}$ em 120 min de tratamento (ponto central do planejamento experimental). Assim, verificou-se a potencialidade da reação de Fenton para degradação de matéria orgânica, principalmente dos compostos que fornecem cor e turbidez ao efluente, verificada via avaliação do espectro de absorção.
\end{abstract}

\section{INTRODUÇÃO}

A mandioca contribui para a alimentação de mais de 700 milhões de pessoas e é uma cultura geradora de empregos. Visto que a raiz é consumida na sua forma natural, mas também como farinha e fécula, há o seu processamento. $\mathrm{O}$ processamento de alimentos, em geral é um dos ramos industriais em que se tem maior consumo de água nas diversas etapas de produção. E, por consequência, observa-se a geração de grande volume de resíduos líquidos com elevada carga orgânica (alta Demanda Química e Bioquímica de Oxigênio DQO e DBO), devido sua composição em misturas complexas de poluentes nas mais variadas concentrações (Bautista et al., 2008).

A manipueira é a água de constituição da raiz misturada a água de lavagem que é gerada no momento da prensagem da mandioca para a produção da sua farinha. Os problemas ambientais causados por esse resíduo são devido ao alto valor da sua demanda bioquímica de 
oxigênio (DBO), que varia entre $14000 \mathrm{mg} \mathrm{L}^{-1}$ até $34000 \mathrm{mg} \mathrm{L}^{-1}$, associada ao grande volume produzido, que varia de 300 a 3000 L por tonelada de raízes processadas. Além disso, possui a linamarina, que é um glicosídeo cianogênico, do qual provém o ácido cianídrico, que causa eutrofização dos corpos d'água, morte da fauna aquática e dos animais que consomem a água por ele contaminada (Campos et al., 2006).

A relação denominada de "população equivalente" é quando se compara a DBO proveniente de despejos orgânicos gerados pela indústria com a contribuição normal "per capita" de esgotos domésticos. Sendo assim, considerando o índice equivalente em DBO (5 dias, $20^{\circ} \mathrm{C}$ ) de 54 gramas /habitante/dia, uma fecularia e uma casa de farinha que processem, individualmente, uma tonelada de raízes por dia, equivalem, respectivamente, à poluição causada por 200 a 300 e 150 a 200 habitantes/dia (Fioretto, 1994).

Tendo essa problemática em vista, estudos a respeito das maneiras de remover os contaminantes das águas são necessários. Filtros comuns, floculação, cloração e outras formas de tratamentos convencionais são ineficientes para o tratamento da manipueira. Sendo assim, os Processos Oxidativos Avançados (POAs) se mostram como uma possível alternativa de tratamento. Estes processos são caracterizados pela geração do radical hidroxila $(\bullet \mathrm{OH})$, com potencial padrão de redução de $2,8 \mathrm{~V}$. A reação de oxidação acontece de forma rápida, não seletiva e eficiente, podendo até mesmo mineralizar moléculas orgânicas complexas. Os principais processos de geração de radicais hidroxilas são a fotólise, oxidação com peróxido de hidrogênio, reações de Fenton e foto-Fenton, ozonização, oxidação eletroquímica e a fotocatálise heterogênea (Teixeira; Jardim, 2004). A reação de Fenton foi descoberta por H. J. H. Fenton em 1894 e seu princípio baseia-se que o peróxido de hidrogênio $\left(\mathrm{H}_{2} \mathrm{O}_{2}\right)$ pode ser ativado por íons ferrosos $\left(\mathrm{Fe}^{2+}\right)$ para oxidar moléculas orgânicas. Esse POA foi utilizado com eficiência em tratamento de água residual para a remoção de muitos produtos orgânicos. $\mathrm{O}$ processo de Fenton pode ser realizado a temperatura ambiente e a pressão atmosférica e os reagentes requeridos são disponíveis, fáceis de armazenar e manusear (Babuponnusami; Muthukumar, 2014).

Desta forma, o objetivo deste trabalho foi avaliar as reações de Fenton via espectrofotometria UV-Vis para a degradação da matéria orgânica de manipueira pré-tratada.

\section{MATERIAIS E MÉTODOS}

Os estudos foram realizados utilizando efluente real da produção de fécula de mandioca, obtido em uma unidade de processamento na região norte central do Paraná - Brasil. Para fins de caracterização da amostra, foram determinados: $\mathrm{pH}$ (potenciômetro), turbidez (turbidímetro), condutividade elétrica (condutivímetro), DQO [colorimetria de refluxo fechado segundo descrito em APHA (1998)], COT (analisador de carbono Shimadzu TOC 5000-A), sólidos totais, voláteis, fixos [gravimetria segundo descrito em A.O.A.C. (2000)] e sedimentáveis [método do cone Imhoff segundo NBR 10561 (1988)], acidez volátil/alcalinidade (SILVA, 1981), concentração de ferro total (o-fenantrolina) e fósforo total (APHA, 1998). 
Para tratamento primário da manipueira, foram realizados experimentos de coagulação/floculação com solução de cloreto férrico $\left(\mathrm{FeCl}_{3}\right) 1200 \mathrm{mg} \mathrm{L}^{-1}$ e pH 6,0. Posteriormente, foi realizado um tratamento secundário com lodo ativado em sistema SBR (Sequencing Batch Reactor), em reatores de polipropileno, num sistema com capacidade de tratamento de 5,5 L. As condições da coagulação e do tratamento biológico foram obtidas em experimentos prévios (dados não apresentados). O resíduo foi submetido ao processo tipo Fenton em jar test com $200 \mathrm{~mL}$ de manipueira (Figura 1).

Figura 1 - Jar test utilizado com 6 experimentos diferentes.

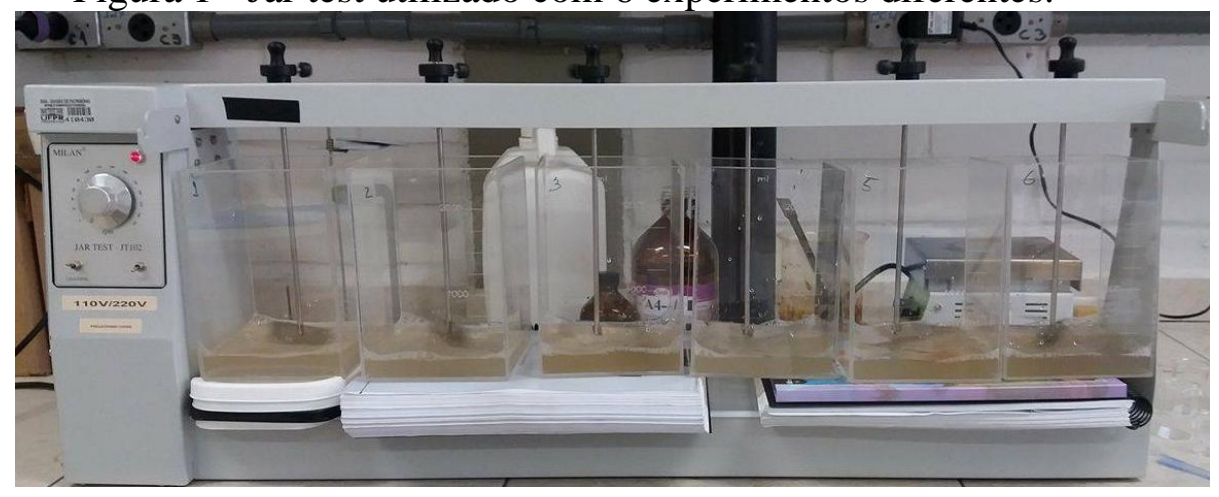

Para adequar as melhores condições do tratamento da manipueira, foi realizado um planejamento experimental $3^{2}$, com quatro repetições no ponto central, variando-se o $\mathrm{pH}$, concentração de $\mathrm{H}_{2} \mathrm{O}_{2}$ e $\mathrm{Fe}^{2+}$, de acordo com a tabela 1 .

Tabela 1 - Planejamento experimental do processo Fenton.

\begin{tabular}{|c|c|c|c|}
\hline Experimento & $\mathrm{pH}$ & {$\left[\mathrm{H}_{2} \mathrm{O}_{2}\right]\left(\mathrm{g} \mathrm{L}^{-1}\right)$} & {$\left[\mathrm{Fe}^{2+}\right]\left(\mathrm{mg} \mathrm{L}^{-1}\right)$} \\
\hline 1 & 3,2 & 15 & 60 \\
\hline 2 & 3,2 & 20 & 300 \\
\hline 3 & 3,0 & 10 & 300 \\
\hline 4 & 2,8 & 20 & 180 \\
\hline 5 & 2,8 & 15 & 300 \\
\hline 6 & 3,0 & 20 & 60 \\
\hline 7 & 3,0 & 15 & 180 \\
\hline 8 & 3,2 & 10 & 180 \\
\hline 9 & 2,8 & 10 & 60 \\
\hline 10 & 3,0 & 15 & 180 \\
\hline 11 & 3,0 & 15 & 180 \\
\hline 12 & 3,0 & 15 & 180 \\
\hline
\end{tabular}

Após o início de cada reação, eram retiradas alíquotas de $1 \mathrm{~mL}$ e realizada análise espectrofotométrica por UV-vis para se observar a variação espectral $(800-200 \mathrm{~nm}) \mathrm{em}$ espectrofotômetro ProAnálise (UV 6100PC), ao longo de 120 minutos de tratamento. Em intervalos pré-estabelecidos, essa operação foi repetida. Para finalizar as reações, o $\mathrm{pH}$ das amostras foi ajustado para 9,0 com solução de $\mathrm{NaOH} 1 \mathrm{~mol} \mathrm{~L}^{-1}$.

\section{RESULTADOS}

Na tabela 2 encontram-se os resultados obtidos na caracterização da manipueira. 
Tabela 2 - Caracterização média da manipueira

\begin{tabular}{|l|c|}
\hline \multicolumn{1}{|c|}{ Parâmetros } & Concentração \\
\hline $\mathrm{pH}$ & $3,74 \pm 0,02$ \\
\hline Turbidez $(\mathrm{NTU})$ & $171 \pm 2$ \\
\hline $\mathrm{DQO}\left(\mathrm{mgO}_{2} \mathrm{~L}^{-1}\right)$ & $16884,44 \pm 1238,88$ \\
\hline $\mathrm{COT}\left(\mathrm{mg} \mathrm{L}^{-1}\right)$ & 2892,4 \\
\hline Sólidos totais $(\%)$ & $0,912 \pm 0,091$ \\
\hline Sólidos voláteis $(\%)$ & $0,247 \pm 0,117$ \\
\hline Sólidos fixos $(\%)$ & $0,665 \pm 0,133$ \\
\hline Sólidos sedimentáveis $\left(\mathrm{mL} \mathrm{L}^{-1}\right)$ & $69 \pm 4$ \\
\hline Condutividade elétrica $\left(\mu \mathrm{S} \mathrm{cm}^{-1}\right)$ & $1793 \pm 1$ \\
\hline Fósforo total $\left(\mathrm{mg} \mathrm{L}^{-1}\right)$ & $3484 \pm 87,02$ \\
\hline Ferro total $\left(\mathrm{mg} \mathrm{L}^{-1}\right)$ & $17,4 \pm 0,4$ \\
\hline Acidez/alcalinidade & $4,29 \pm 0,30$ \\
\hline
\end{tabular}

Para análise da eficiência da reação, foram feitas varreduras espectrais (Figura 2 e 3).

Figura 2 - Espectros de absorção para (a) pH 3,2; $\left[\mathrm{H}_{2} \mathrm{O}_{2}\right]=15 \mathrm{~g} \mathrm{~L}^{-1} \mathrm{e}\left[\mathrm{Fe}^{2+}\right]=60 \mathrm{mg} \mathrm{L}{ }^{-1}$; (b) $\mathrm{pH} \mathrm{3,2;}\left[\mathrm{H}_{2} \mathrm{O}_{2}\right]=20 \mathrm{~g} \mathrm{~L}^{-1}$ e $\left[\mathrm{Fe}^{2+}\right]=300 \mathrm{mg} \mathrm{L}^{-1}$; (c) $\mathrm{pH} \mathrm{3,0;}\left[\mathrm{H}_{2} \mathrm{O}_{2}\right]=10 \mathrm{~g} \mathrm{~L}^{-1}$ e $\left[\mathrm{Fe}^{2+}\right]=300$

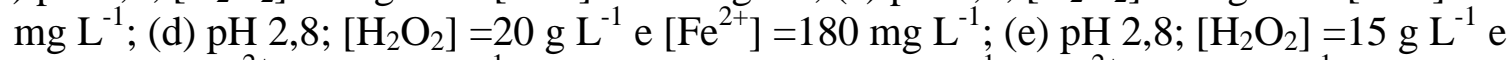
$\left[\mathrm{Fe}^{2+}\right]=300 \mathrm{mg} \mathrm{L}^{-1}$; (f) $\mathrm{pH} 3,0 ;\left[\mathrm{H}_{2} \mathrm{O}_{2}\right]=20 \mathrm{~g} \mathrm{~L}^{-1}$ e $\left[\mathrm{Fe}^{2+}\right]=60 \mathrm{mg} \mathrm{L}^{-1}$

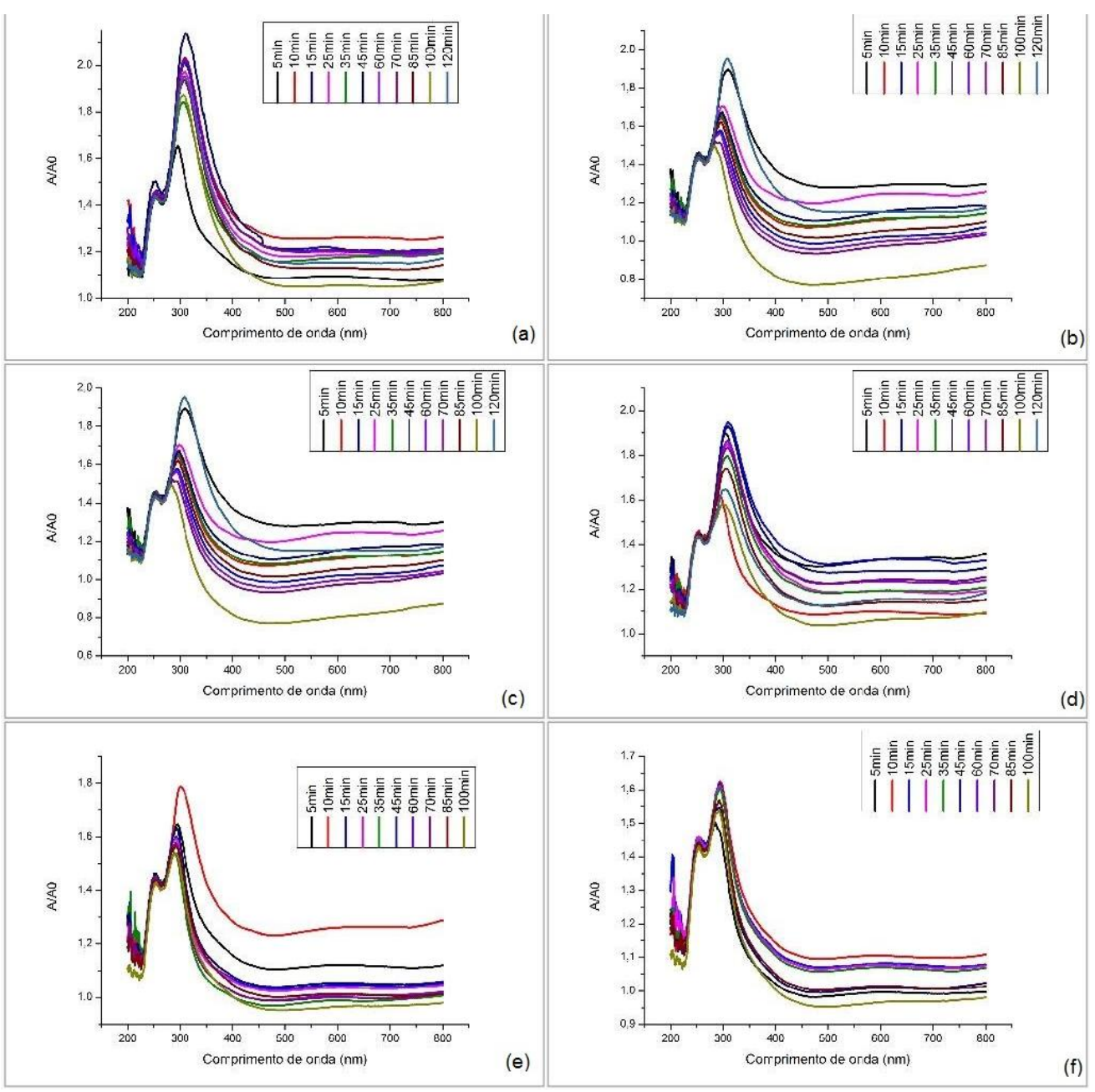


Figura 3 - Espectros de absorção para (g) pH 3,0; $\left[\mathrm{H}_{2} \mathrm{O}_{2}\right]=15 \mathrm{~g} \mathrm{~L}^{-1} \mathrm{e}\left[\mathrm{Fe}^{2+}\right]=180 \mathrm{mg} \mathrm{L}^{-1}$; (h) $\mathrm{pH} 3,2 ;\left[\mathrm{H}_{2} \mathrm{O}_{2}\right]=10 \mathrm{~g} \mathrm{~L}^{-1}$ e $\left[\mathrm{Fe}^{2+}\right]=180 \mathrm{mg} \mathrm{L}^{-1}$; (i) $\mathrm{pH} 2,8 ;\left[\mathrm{H}_{2} \mathrm{O}_{2}\right]=10 \mathrm{~g} \mathrm{~L}^{-1}$ e $\left[\mathrm{Fe}^{2+}\right]=60 \mathrm{mg}$ $\mathrm{L}^{-1}$; (j) $\mathrm{pH} 3,0 ;\left[\mathrm{H}_{2} \mathrm{O}_{2}\right]=15 \mathrm{~g} \mathrm{~L}^{-1}$ e $\left[\mathrm{Fe}^{2+}\right]=180 \mathrm{mg} \mathrm{L}^{-1}$; (k) pH 3,0; $\left[\mathrm{H}_{2} \mathrm{O}_{2}\right]=15 \mathrm{~g} \mathrm{~L}^{-1}$ e $\left[\mathrm{Fe}^{2+}\right]$ $=180 \mathrm{mg} \mathrm{L}^{-1}$; (l) $\mathrm{pH} 3,0 ;\left[\mathrm{H}_{2} \mathrm{O}_{2}\right]=15 \mathrm{~g} \mathrm{~L}^{-1} \mathrm{e}\left[\mathrm{Fe}^{2+}\right]=180 \mathrm{mg} \mathrm{L}^{-1}$

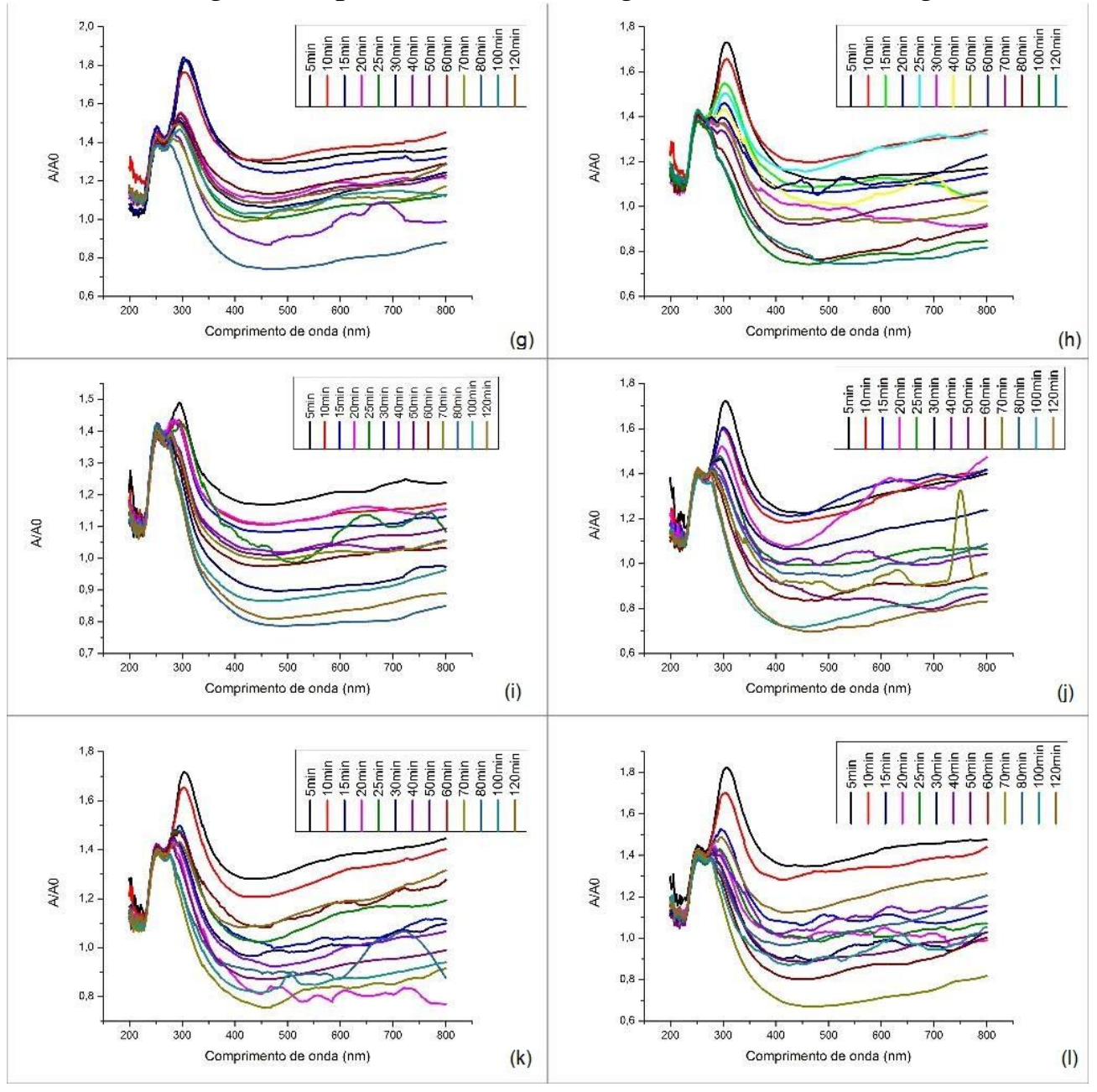

Pode-se observar que na sequência de reações, a relação A/A0 foi reduzida com o decorrer dos processos. Algumas condições favoreceram o decaimento no parâmetro: $\mathrm{pH}$ 3,0, $\left[\mathrm{H}_{2} \mathrm{O}_{2}\right]$ de $15 \mathrm{~g} \mathrm{~L}^{-1}$ e Fe $\mathrm{Fe}^{2+} 180 \mathrm{mg} \mathrm{L}^{-1}$ em 120 min [Fig. 3(G), (J), (K), (L); pH 3,0, $\left[\mathrm{H}_{2} \mathrm{O}_{2}\right]$ de $10 \mathrm{~g} \mathrm{~L}^{-1}$ e Fe $^{2+} 300 \mathrm{mg} \mathrm{L}^{-1}$ em 120 min [Fig. 2 (C)]. Em outras condições, as relações foram mais altas e tempos mais curtos seriam suficientes para melhoria do parâmetro em questão. A possível explicação para este resultado é que, no início da reação, os compostos facilmente oxidáveis são removidos. Como a oxidação continua, estes compostos se tornam menos disponíveis e alguns intermediários gerados tornam-se sequestradores de radicais hidroxilas, competindo radicais com moléculas não degradadas (Guzmán et al., 2016).

\section{CONCLUSÃO}

No tratamento de efluentes por Processos Oxidativos Avançados, a formação de radicais dependerá do $\mathrm{pH}$, presença de íons e do tipo de poluente em questão, e a taxa de 
oxidação irá variar conforme as concentrações de radical, oxigênio e do poluente. Por isso, foram estudadas diferentes condições para a reação de Fenton em manipueira, a fim de encontrar condições mais adequadas para degradação deste subproduto. Um controle analítico simples é pela varredura do espectro de absorção da amostra frente aos diversos tratamentos, em que o parâmetro $\mathrm{A} / \mathrm{A}_{0}$ fornece um indicativo de conversão de moléculas orgânicas complexas (contaminantes) em produtos mais simples, condição para que um tratamento seja definido como POA. Desta forma, a condição encontrada que alcançou melhores taxas foi em $\mathrm{pH}$ reacional de 3,0, $\left[\mathrm{H}_{2} \mathrm{O}_{2}\right]$ de $15 \mathrm{~g} \mathrm{~L}^{-1}$ e $\mathrm{Fe}^{2+} 180 \mathrm{mg} \mathrm{L}^{-1}$ em $120 \mathrm{~min}$ de experimento.

\section{REFERÊNCIAS}

ABNT (Associação Brasileira e Normas Técnicas): NBR 10561. Águas - Determinação de resíduo sedimentável (sólidos sedimentáveis) - Método do cone de Imhoff. Rio de Janeiro, 1988. 2 p.

APHA (1998). Standard methods for the examination of water and wastewater. 20a edição. Washington:APHA/AWWA.

Babuponnusami, A.; Muthukumar, K. A. A review on Fenton and improvements to the Fenton process for wastewater treatment. J. Environ. Chem. Eng., v. 2, p. 557-572, 2014.

Bautista, P.; Mohedano, A. F.; Casas, J. A.; Zazo, J. A.; Rodriguez, J. J. An overview of the application of Fenton oxidation to industrial wastewaters treatment. J. Chem. Tech. Biotechnol., v. 83, p. $1323-1338,2008$.

Campos, A. T.; Daga, J.; Rodrigues, E. E.; Franzener, G.; Suguiy, M. M. T.; Syperreck, V. L. G. Tratamento de águas residuárias de fecularia por meio de lagoas de estabilização. Eng. Agríc., v. 26, n. 1, p. 235-242, 2006.

Fioretto, R. A. Uso direto da manipueira em fertirrigação. In: Cereda, M. P. Industrialização da mandioca no Brasil. São Paulo: Paulicéia, p.51-80, 1994.

Guzmán, J.; Mosteo, R.; Sarasa, J.; Alba, J. A.; Ovelleiro, J. L. Evaluation of solar photo-Fenton and ozone based processes as citrus wastewater pre-treatments. Sep. Purif. Technol., v. 164, p. 155-162, 2016.

Silva, D. J. Análise de alimentos - métodos químicos e biológicos. Viçosa: UFV - Imprensa Universitária, 1981.

Teixeira, C. P. A. B.; Jardim, W. F. Caderno temático: Processos oxidativos avançados - Conceitos teóricos. Instituto de Química. Universidade Estadual de Campinas - UNICAMP - Campinas, 2004. 